\title{
Implementación de un programa público de salud mental escolar en Chile en el contexto de la nueva gestión pública
}

\author{
Implementation of a School Mental Health Program in Chile \\ in the Context of New Public Management
}

\begin{abstract}
Gloria Zavala-Villalón, Catalina Ganga-León, Rodrigo Rojas-Andrade, Betzabé Torres Cortés y Loreto Leiva Bahamondes*
\end{abstract}

\begin{abstract}
Resumen: Esta investigación tiene como objetivo caracterizar la implementación de un programa público de salud mental escolar a través de las prácticas y perspectivas de quienes lo ejecutan, bajo el prisma de la nueva gestión pública en Chile. Por medio de análisis de contenido cualitativo de nueve grupos focales y cuatro entrevistas, los resultados evidenciaron que la implementación se caracteriza por ejecutores cuyas prácticas están fuertemente influidas por sus años de experiencia y por una ejecución que requiere altos niveles de adaptabilidad y que va más allá de lo indicado en las orientaciones técnicas. Se discuten sus implicancias a la luz de la forma que ha tomado la descentralización en América Latina y sus consecuencias para las políticas públicas.
\end{abstract}

* Gloria Zavala-Villalón es profesora asistente del Departamento de Psicología, Facultad de Ciencias Sociales (Facso), Universidad de Chile. Avenida Capitán Ignacio Carrera Pinto 1045, Nuñoa, Santiago, Chile. Tel: +5622 9787 2409. Correo-e: gzavala@uchile.cl. oncid: https://orcid.org/0000-0002-37324602. Catalina Ganga-León es asistente de investigación Proyecto Fondecyt Regular núm. 1171634, Departamento de Psicología, Facso, Universidad de Chile. Avenida Capitán Ignacio Carrera Pinto 1045, Nuñoa, Santiago, Chile. Tel: +5622 9787 2409. Correo-e: cgleon@ug.uchile.cl. orcid: https://orcid.org/00000002-3054-4522. Rodrigo Rojas-Andrade es profesor asociado, Escuela de Psicología, Universidad Academia de Humanismo Cristiano. Condell 343, Providencia, Santiago, Chile. Tel: +5622 2787 8100. Correo-e: rrojasa01@docentes.academia.cl. orciD: https:/orcid.org/0000-0002-6459-6902. Betzabé Torres Cortés, tesista de magíster Proyecto Fondecyt Regular núm. 1171634, Departamento de Psicología, Universidad de Chile. Avenida Capitán Ignacio Carrera Pinto 1045, Nuñoa, Santiago, Chile. Tel: +5622 97872404. Correo-e: betzabe.torres@ug.uchile.cl. orciD: https://orcid.org/0000-0001-6815-4186. Loreto Leiva Bahamondes, profesora asociada, Departamento de Psicología, Facso, Universidad de Chile. Avenida Capitán Ignacio Carrera Pinto 1045, Nuñoa, Santiago, Chile. Tel: +562297872404, Correo-e: loretoleivab@u. uchile.cl. ORCID: https://orcid.org/0000-0003-0837-3699. Este artículo es parte del Proyecto Fondecyt Regular núm. 1171634 "La implementación como elemento clave en el éxito de una intervención en salud mental escolar: Un estudio mixto de los dominios generales y las dimensiones locales involucrados". Comisión Nacional de Investigación Científica y Tecnológica (Conicyt), Ministerio de Educación, Chile.

Artículo recibido el 22 de enero de 2019 y aceptado para su publicación el 5 de marzo 2020.

Dor: http://dx.doi.org/10.29265/gypp.v29i2.780 
Palabras clave: nueva gestión pública, descentralización, implementación, programas gubernamentales, programas de salud mental escolar.

Abstract: This research aimed to characterize the implementation of a public school mental health program through the practices and perspectives of those who execute it under the prism of the New Public Management in Chile. Through qualitative content analysis of nine focus groups and four interviews, the results showed that the implementation is characterized by implementers whose practices are strongly influenced by their years of experience, and for an execution that requires high levels of adaptability and goes beyond what is indicated in the technical guidelines. Its implications are discussed given the way decentralization has taken in Latin America and its consequences for public policies.

Keywords: new public management, decentralization, implementation, government programs, school mental health programs.

Fin n las últimas décadas, la nueva gestión pública (NGP) ha jugado un rol central entre otros fines, hacia estándares y medidas de desempeño, control de resultados, y descentralización de las unidades del sector público (Hood, 1991). Estos principios tienen repercusiones relevantes para la implementación de programas, que van desde la facilitación de su ejecución hasta la obstaculización.

La NGP hace referencia a una serie de reformas para la evaluación y rediseño del sector público, que surgen en países anglosajones a principios de la década de 1980 en respuesta a las deficiencias de la administración burocrática y siguiendo las influencias de organismos tales como el Banco Mundial (Bм), el Banco Interamericano de Desarrollo (BID), el Fondo Monetario Internacional (FMI) y la Organización para la Cooperación y el Desarrollo Económicos (OCDE), para su implantación en el resto del mundo (Aberbach y Christensen, 2001; Barzelay, 2003; Bryson et al., 2014; Chica y Salazar, 2016; Christensen y Lægreid, 2005; Milanesi y Ramos, 2017; Verbeeten y Speklé, 2015).

Estas reformas se desarrollaron ampliamente entre 1980 y 2010, enfocándose inicialmente en la reducción del Estado a través de descentralización, privatizaciones y externalización de funciones (Christensen y Lægreid, 2005; Guerrero, 2019; Milanesi y Ramos, 2017). La propuesta fue la incorporación de herramientas y lógicas de la gestión privada en el ámbito público, bajo la premisa de que éstas eran racionalmente superiores, con foco en la eficiencia en el aparato estatal (Christensen y Lægreid, 2010; Guerrero, 2019; Milanesi y Ramos, 2017).

Al principio, el foco de la NGP estuvo puesto en las llamadas reformas de primera generación, orientadas a la consecución de un Estado mínimo a través 
de políticas de desregulación, descentralización, privatización, externalización y reducción de dotación de personal. Estas reformas en América Latina, si bien lograron reducir el déficit fiscal, no permitieron mejorar el bajo crecimiento económico de los países de la región (Milanesi y Ramos, 2017).

Posteriormente, las reformas de segunda generación se enfocaron en el fortalecimiento de las capacidades institucionales, haciendo referencia a la "reinvención" del rol del sector público, con fuerte influencia de los desarrollos planteados por Osborne y Gaebler (1994), quienes abogaban por un gobierno empresarial, dirigido a incentivar la generación de recursos. Utilizando los mecanismos del mercado para ofrecer servicios públicos y transformando su rol de proveedor a facilitador de iniciativas de sectores privados, comunitarios y las organizaciones de la sociedad civil (osC), a fin de resolver problemas sociales, separando la formulación de políticas de la prestación directa de los servicios (Osborne y Gaebler, 1994).

En el escenario planteado, los gobiernos delegan poder decisional a unidades y funcionarios de niveles más bajos, separan decisiones estratégicas de operativas, y sustituyen el control burocrático por la supervisión y el monitoreo (Milanesi y Ramos, 2017; Osborne y Gaebler, 1994, Ramos y Milanesi, 2018).

Uno de los elementos nucleares que se distingue en estas reformas es la descentralización, referida a la intervención en la estructura político-administrativa que busca modificar la forma y grado de regulación del Estado en determinados ámbitos políticos (Haefner, 2000). Ésta permite la creación de una serie de nuevos organismos autónomos para ejercer el poder delegado desde el nivel central, con el objetivo de llevar los servicios públicos a un funcionamiento más eficiente, conjuntamente con entregarles mayor capacidad de respuesta y de adaptación a las necesidades locales (Pollitt y Bouckaert, 2011).

Lo expuesto implica que la autoridad decisional se delega de niveles superiores a niveles más bajos de la estructura pública, pero el nivel central concentra la labor estratégica y los organismos autónomos se enfocan en la especialización, permitiéndole al Estado la realización de tareas que le son específicas. Es parte de este tipo de descentralización, la realizada a través de gobiernos territoriales, como municipalidades u otros (Christensen et al., 2007; Pollitt y Bouckaert, 2011).

Esta manera de gestión influye en la estructura y el funcionamiento del sector público, principalmente en lo referido a la fragmentación de la estructura estatal, con disminución de la coordinación tanto vertical como horizontal. Esto conlleva situaciones en que políticas diferentes se socavan entre sí, mecanismos de control limitan la autonomía, y se segmenta en el acceso a servicios por parte de los ciudadanos (Christensen y Lægreid, 2005, 2007, 2008; Diefenbach, 2009). 
Igualmente, es posible observar efectos positivos de la descentralización, pues aleja la función de implementación de programas de la burocracia y entrega un mayor grado de decisión a los que se encuentran en niveles más bajos, introduciendo incentivos para asegurar una implementación eficiente (Denhardt y Denhardt, 2007; Osborne y Gaebler, 1994). Por lo tanto, existe capacidad de responder rápidamente al contexto cambiante y a las necesidades de los clientes o beneficiarios, en tanto son estos trabajadores quienes conocen lo que sucede día a día, y pueden plantear soluciones mejores e innovadoras, al desarrollar más compromiso y productividad gracias a su mayor nivel de participación (Osborne y Gaebler, 1994).

Dado lo expuesto, no es de extrañar que los resultados de los estudios sean contradictorios. Hay investigaciones que señalan que la descentralización, a través de la designación de una entidad autónoma, puede mejorar la productividad en agencias federales u hospitales (Bilodeau et al., 2006; González y Trujillo, 2008; Lee et al., 2008). Por el contrario, se menciona que distribuir las responsabilidades a unidades geográficas más pequeñas se asocia a una menor eficiencia en la gestión (Boyne, 1996). A esto se suma una gran heterogeneidad en cuanto a recursos y capacidades de gestión y administración de los servicios y bienes locales (Leyton, 2006). De hecho, el estudio llevado a cabo por Waissbluth y Arredondo (2011) menciona que las municipalidades (gobiernos territoriales autónomos) "son responsables por 75 diferentes tipos de servicios, son el front office de la ciudadanía, y si bien tienen más atribuciones en lo político, sus recursos humanos y financieros son insuficientes" (Waissbluth y Arredondo, 2011: 4).

Esto da cuenta de que el proceso descentralizador no ha generado una mirada territorial continua, y se observan contradicciones entre los intereses políticos y técnicos sectoriales. En este contexto, se construyen programas cuyo diseño proviene del nivel central, pero su aplicación cae en manos de entes locales descentralizados del estado — municipalidades - u organizaciones del sector privado, los que además no cuentan con suficientes recursos. En América Latina, esto ha provocado problemas en la coordinación de las distintas unidades de toma de decisiones (Haefner, 2000) y dificultades de los programas públicos para cumplir con sus objetivos (Salamon, 2002).

Ejemplo de ello es el desarrollo de la política pública de infancia y adolescencia en Colombia, que implicó la transferencia de la responsabilidad del diseño, ejecución y evaluación de programas y proyectos, tanto a entes del nivel nacional como territoriales. Así, las alcaldías municipales tuvieron un rol fundamental en 
la territorialización de esta política, y fue el Sistema Nacional de Bienestar Familiar el encargado de articular este proceso. Este tipo de estructura multinivel significó una dificultad en la articulación de los distintos actores, con bajos niveles de contribución y eficacia (Pineda y Orduz, 2019).

Algo similar sucedió en México con la política social para combatir la pobreza, cuyo diseño buscaba facilitar la rendición de cuentas a través de la asignación de tareas a responsables específicos, y desagregar los problemas complejos mediante su atención por parte de diversos actores. Ésta contaba con al menos cuatro programas para la mejora de la vivienda de la población en pobreza extrema. Cada uno de ellos entregaba un apoyo específico (por ejemplo, techo, piso o muro) a personas diversas, lo que llevó a la ineficiencia producto de una carencia de integralidad y por categorizaciones no excluyentes, a partir de las cuales una misma población fue objetivo de muchas acciones (Cejudo, 2016).

Sin embargo, la experiencia de México también mostró aciertos, al entregar la oportunidad a los gobernantes locales de ejercer liderazgo. Además, incorporó a líderes comunitarios y organizaciones de la sociedad civil, lo que fue clave para garantizar la participación a través del trabajo en red (Pineda y Orduz, 2019).

En el caso de Chile, el proceso de descentralización del Estado se inició durante la década de 1980, profundizándose a partir de la década de 1990, con énfasis en el ámbito político y administrativo (Raczynski, 1994). Entre otras cosas, impulsó la generación de gobiernos territoriales autónomos — las municipalidades-, cuya característica es la gestión de una parte del territorio con recursos propios. Así, los municipios, como gobiernos locales, se hicieron cargo —en muchos casos- de la ejecución e implementación de programas sociales diseñados por el gobierno central, pero ejecutados a nivel local, ejemplo de ello es el programa de salud mental en la escuela, objeto de este estudio.

Otro de los elementos nucleares identificados en estas reformas y en específico en las reformas de segunda generación en América Latina fue el enfoque de gestión por resultados, con énfasis en el logro de objetivos y metas, que dirige a los actores hacia la creación de valor público mediante instrumentos de verificación de avances e indicadores operacionales (insumos), de gestión (medios) y de resultados (fines) (Chica, 2011; Chica y Salazar 2016).

Esta gestión por resultados o instrumentos de cálculo se configuró con la finalidad de estandarizar el sector público, facilitar la toma de decisiones, permitir la evaluación pública y proyectar acciones y recursos (Miller, 2001), con el fin de generar representaciones comunes entre los actores respecto de metas y relaciones (Klijn et al., 2010; Lester y Reckhow, 2013), en conjunto con simplificar procesos 
de trabajo multidimensionales y complejos, convirtiéndolos en indicadores manejables que permitiesen la comparación entre procesos diversos desde una mirada homogénea.

$\mathrm{Al}$ respecto, Pollitt (2003) plantea que centrarse en indicadores implica una sobreconcentración en lo cuantificable (costos, número de usuarios atendidos, etc.) y una subconcentración en aspectos que no son fácilmente medibles. Así, este tipo de gestión se ajusta a prácticas canónicas en las que los problemas son conocidos y los estándares compartidos, pero no lo hace frente a prácticas no canónicas y aquellas en transición en las que no existen consensos previos. En lugar de datos, estas últimas requieren diálogo para gestionar la ambigüedad y los espacios interpretativos (Noordegraaf y Abma, 2003).

Soto y colaboradores (2017), en su estudio sobre instrumentos en redes de acción pública, identifican frecuentes tensiones entre calidad y cantidad de actividad, en cuanto a temporalidades de la acción, prioridades y cargas de trabajo. En la misma línea, destacan la necesidad de los actores de ampliar su campo de acción, más allá de las restricciones dadas por los instrumentos, en función de las características locales, mostrando que "se suele arrastrar una sensación que hay algo fundamental del propósito de los programas que no se está logrando, ya que las acciones dentro de los instrumentos son insuficientes" (Soto et al., 2017: 84).

De esta forma, el foco en objetivos cuantificables, basados en valores como la eficiencia y el desempeño, implican dificultades para los trabajadores públicos, que se ven obligados a dejar de lado valores compartidos de servicio, para concentrarse en la lógica económica de la gestión para resultados (Emery y Giauque, 2003; Hood, 1991; Pollitt, 2003).

Estos antecedentes llevan a cuestionarse acerca del rol de la NGP y en específico de la descentralización y la gestión por resultados en la implementación de programas, que pueden actuar como facilitadores o barreras frente a la puesta en práctica de una intervención. Por un lado, promueven y permiten una mayor flexibilidad, innovación, compromiso y productividad, lo cual estimula la autonomía y participación (Osborne y Gaebler, 1994); por otro, corren el riesgo de generar una fragmentación de la administración y dificultades de coordinación y cooperación entre las entidades (Pérez et al., 2011), limitando la capacidad de respuesta a las necesidades que emergen a nivel local. En efecto, al ser uno de los focos de la NGP, la reducción de la complejidad y la heterogeneidad de la práctica (Soto et al., 2017), se desvía la atención hacia una gestión de indicadores, lo que conlleva el riesgo de no considerar ni las particularidades de cada práctica ni sus efectos sociales (Cunill, 2009). 
Dadas las dificultades identificadas en la NGP surgen una serie de reformas orientadas hacia mecanismos de solución de problemas públicos en formas colaborativas, bajo el nombre de post-nueva gestión pública (PNGP) (Christensen y Fan, 2018; Van Gestel y Teelken, 2004). Ésta busca la cooperación mediante la recentralización para mejorar la coordinación, responsabilidad y transparencia (Christensen y Lægreid, 2005, 2007, 2008; Dunleavy et al., 2011; Pérez et al., 2011; Stoker, 2006); además, rescata valores de equidad social y humanización de las políticas.

No obstante, y a pesar de que la PNGP deja atrás la descentralización y la delegación de autoridad por un foco en lo social y organizacional en cuanto a interrelaciones y coordinación vertical y horizontal de la administración pública (Christensen y Lægreid, 2005, 2007), la gestión pública continúa principalmente bajo la influencia de la NGP. Esto significa que los programas se diseñan a nivel gubernamental o central pero se implementan a nivel local, y son los organismos de este último nivel los encargados de implementar políticas y programas públicos, los cuales no necesariamente han sido diseñados para su ejecución en contextos locales específicos.

Ante este panorama es posible exponer que la implementación de programas - entendida como el "proceso de poner en marcha una práctica o programa en el funcionamiento de una organización, como una escuela, y que puede ser visto como una serie de actividades diseñadas para lograrlo" (Forman et al., 2013: 78) —, implica variabilidad en su aplicación, lo cual tensiona su ejecución.

La literatura sobre implementación se concentra principalmente en países angloparlantes (Barry et al., 2013; Atilola y Ola; 2016), cuyas realidades políticas y administrativas difieren de las latinoaméricanas y chilena en particular, ya que la implementación de las intervenciones no se encuentra mediada por la compleja red de actores que caracteriza la descentralización estatal, sino que opera con menos mediadores administrativos y con una menor variabilidad respecto de los recursos.

Lo anterior revela la necesidad de investigar la temática en regiones como América Latina, donde la realidad difiere en los distintos niveles contextuales y donde no necesariamente se consideran las diferencias de los resultados reportados según el contexto (Fazel et al., 2014) y tampoco se asume la brecha entre lo diseñado a nivel central y lo ejecutado a nivel local. Así, el estudio de la implementación que integre el nivel contextual, en este caso, desde las reformas de la NGP mencionadas, permitirá una mejor descripción de los resultados obtenidos en cada realidad local. 
De esta forma, y entendiendo que estos aspectos pueden operar como facilitadores o barreras, este estudio tiene como propósito caracterizar la implementación de un programa público de salud mental escolar (sME) a través de las prácticas y perspectivas de quienes lo ejecutan, en el contexto de la NGP y la descentralización en Chile, a fin de identificar sus implicaciones en la implementación de un programa nacional de SME inserto en el gobierno local y según un modelo de gestión para resultados (Miller, 2001). Además contribuirá al estudio de la aplicación de programas y políticas en contextos diversos, al considerar al hecho de que la gran mayoría de los estudios sobre implementación de la NGP se han llevado a cabo en los contextos en los cuales se originó.

\section{MÉTODO}

\section{Tipo de estudio}

Se utilizó una metodología de carácter cualitativo, con el objetivo de caracterizar la implementación de un programa público de SME, desde las prácticas y perspectiva de los actores involucrados, dando cuenta de las formas simbólicas que median y configuran las experiencias sociales, a fin de indagar el fenómeno desde la perspectiva de los actores involucrados (Norẽa et al., 2012; Vain, 2012). El uso de este tipo de metodología se orientó a indagar la posición de los trabajadores (ejecutores) que implementan el programa desde las decisiones operativas, con base en los problemas y desafíos que enfrentan en la puesta en práctica de la intervención. El diseño de la investigación fue descriptivo, pues buscó definir, categorizar y caracterizar elementos relevantes para describir el fenómeno estudiado (Ato et al., 2013)."

\section{Características del programa estudiado}

El programa público estudiado es un modelo de intervención psicosocial que incorpora acciones de detección y prevención del riesgo, promueve estrategias de autocuidado y desarrolla habilidades para la convivencia de los diferentes actores de la comunidad escolar. Ha sido implementado en escuelas municipales y particulares subvencionadas chilenas desde el año 1998 por un organismo dependiente del Ministerio de Educación.

Administrativamente se compone de tres niveles de gestión que implican la interacción entre el organismo dependiente del Ministerio de Educación y los gobiernos locales comunales (municipalidades). El primer nivel corresponde a la Dirección Nacional de dicho organismo, encargada del diseño, monitoreo, evaluación y control del programa a nivel nacional, según la estrategia de intervención 
planeada, asegurando vigilancia técnica y administrativa. El segundo nivel se compone de su Dirección Regional, cuya función es supervisar, hacer seguimiento y acompañar, en sus aspectos administrativos y técnicos, a las entidades ejecutoras o comunales del programa por medio de coordinadores regionales. Por último, el tercer nivel corresponde al comunal, es decir, las municipalidades o gobiernos locales, encargados de coordinar acciones con las escuelas, sus autoridades y otros actores de la red local, siendo responsable del cumplimiento de la administración del equipo técnico del programa.

En el último nivel se insertan los equipos ejecutores locales, que implementan el programa en las escuelas, dependen administrativamente de la municipalidad y técnicamente, de las direcciones regionales. Los equipos cuentan con un coordinador por comuna, que puede ser uno de los profesionales interventores o un gestor comunal. Los equipos fluctúan entre tres y doce personas aproximadamente, dependiendo del tamańo de la comuna, su presupuesto y de la cantidad de escuelas atendidas.

Los municipios interesados en el programa postulan para ejecutarlo en su comuna, y deben aportar al menos 20 por ciento de la inversión anual en caso de ser seleccionadas, además de entregar cartas de compromiso firmadas por los directores de las escuelas participantes. De este modo, la implementación del programa se produce, sobre todo, por agencias municipales, identificándose tres tipos de sectores: educación, salud y servicios sociales comunitarios. También existen, en menor medida, universidades y sectores gubernamentales a cargo del programa (Junaeb, 2017).

Cabe mencionar que la implementación del programa de SME estudiado implica un compromiso de corresponsabilidad tanto con los municipios como con las escuelas, en donde la política pública supone interés y voluntariedad en la participación.

Igualmente, es posible encontrar tres instrumentos de cálculo en el programa, que dan cuenta de las metas anuales y de su cumplimiento, tanto en términos de cantidad de actividades cubiertas, como de ejecución presupuestaria. Estos instrumentos son una programación anual de actividades, a nivel comunal, en donde se estipula tanto el plan de trabajo como la cobertura y el presupuesto; un informe de avance, a mediados de ańo, que da cuenta del progreso y potenciales cambios a la fecha, y un informe final, que implica el cierre de la intervención anual, explicitando las actividades logradas y la ejecución presupuestaria. Además, el Manual de Orientaciones Técnico-metodológicas aparece como un instrumento de orientación estratégica que enmarca, a modo de directriz, la intervención y las bases que sustentan sus objetivos. 
Dado lo expuesto, el contexto chileno aparece como un aporte diferenciador, pues permite estudiar la implementación en escenarios públicos más complejos (Rojas-Andrade et al., 2017), así como también investigar programas de SME desde el campo de la implementación, focalizándose no sólo en la eficacia de este tipo de intervenciones (Silverman y Hinshaw, 2008; Sarno et al., 2014), sino también enfatizar el rol que cumplen los factores contextuales en su éxito o fracaso (Leiva et al., 2020).

\section{Técnica de recolección de datos}

Se utilizaron dos técnicas: a) el grupo focal, útil al investigar relatos comunes, con la finalidad de encontrar la "experiencia" típica de un grupo social. Éste facilita que un pequeño grupo de personas aborde un tema específico, permite la interacción entre los participantes y ofrece flexibilidad para explorar nuevos aspectos (Flick, 2007). En concreto, los grupos focales realizados buscaron explorar y discutir el uso de las orientaciones técnico-metodológicas (manual) por parte de los equipos ejecutores en su cotidianidad. b) La entrevista semiestructurada, que busca producir un discurso conversacional con cierta línea argumental, cuyo grado intermedio de espontaneidad se refiere al nivel de estructuración previa de las preguntas y respuestas (Marradi et al., 2007), las cuales exploraron las percepciones de los coordinadores regionales respecto a los aspectos administrativos y estructurales de la intervención en sus realidades locales.

\section{Participantes}

Primero, los datos fueron producidos a través de nueve grupos focales. Cada grupo focal estuvo compuesto por profesionales ejecutores encargados de la implementación de la intervención en salud mental escolar, quienes debían tener al menos dos años de antigüedad en el cargo con la finalidad de contar con relatos de más experiencias con el programa estudiado. De este modo, se contó con la participación de 87 ejecutores de diez regiones de Chile, a saber, II, III, IV, V, VI, VII, VIII, IX, XII y XV. De los 87 participantes, 65 correspondía a profesionales mujeres. En su mayoría, los participantes eran profesionales de las áreas de ciencias sociales, educación y de la salud. Cada grupo focal regional contó con profesionales de diferentes comunas dentro de dicha región, lo que generaba mayor variabilidad en las experiencias no sólo a lo largo del país, sino también dentro de las mismas regiones. Los ejecutores trabajaban en su mayoría con escuelas municipales, con un índice de vulnerabilidad escolar elevado (IVE). El IVE es construido con variables asociadas a la condición socioeconómica de quienes 
ingresan al sistema escolar financiado por el Estado (Leiva et al., 2015). Además, estas escuelas estaban situadas en comunas con mayores niveles de vulnerabilidad social y menos recursos en términos materiales o de infraestructura.

Los grupos focales fueron realizados entre los años 2016 y 2017, y los moderadores utilizaron una pauta semiestructurada orientada a discutir la opinión de los ejecutores sobre el uso de las orientaciones técnico-metodológicas, qué utilidad le adjudicaban al manual en términos teóricos y prácticos, cómo evaluaban la intervención y su implementación, y de qué modo esto se traducía en su vivencia como trabajadores de un programa público de salud mental escolar.

Además, se trabajó con entrevistas semiestructuradas realizadas a cuatro coordinadores regionales de la intervención, pertenecientes a las regiones de la zona centro del país. Los coordinadores regionales fueron mayoritariamente psicólogos o trabajadores sociales, con alrededor de trece a quince ańos de experiencia en el cargo.

Las entrevistas fueron realizadas durante junio y agosto de 2018, y se trabajó con una pauta temática, cuya finalidad fue explorar los aspectos administrativos y estructurales de la intervención en SME estudiada desde la posición de los coordinadores regionales, así como sus impresiones respecto al trabajo realizado por los equipos ejecutores que tenían a su cargo.

\section{Estrategia de análisis}

Se realizó un análisis de contenido convencional de datos cualitativos, por medio de un proceso de identificación, codificación y clasificación de ciertas temáticas o patrones emergentes del texto (Hsieh y Shannon, 2005). Estas categorías emergentes se centraron en describir las características de la implementación, tanto en términos de la gestión político-administrativa como respecto de las prácticas cotidianas de los equipos ejecutores del programa. De este modo, de acuerdo con su naturaleza cualitativa, la investigación se realizó en un proceso "circular", volviendo tanto a los hechos y luego a su interpretación, como a la inversa (Canales, 2006).

Con la finalidad de dar validez interna a los resultados, inicialmente los grupos focales y las entrevistas semiestructuradas fueron transcritos y leídos por todo el equipo de investigación, para tener una impresión global de los datos. Luego, los textos fueron codificados y categorizados con el software ATLAS.ti 8.0. Después, se procedió a generar interpretaciones de la información subyacente al relato, generando categorías emergentes, las cuales se presentan en el apartado de resultados.

Dado que lo que se pretende es identificar desde el discurso de los propios ejecutores aquellos elementos que caracterizan la implementación de un programa en SME, con foco en el significado y la interpretación subjetiva del contenido 
de los datos conversacionales, lo que se identifica y establece son los contenidos centrales para responder al objeto de estudio, pero no se utilizan variables en el sentido tradicional de éstas, pues ni conceptual ni operativamente son pertinentes para la metodología y el tipo de análisis efectuado.

\section{Aspectos éticos}

La investigación fue aprobada y respaldada por las autoridades nacionales de la intervención en sME analizada y contó con la aprobación del Comité de Ética de la Investigación de la Universidad de Chile, del cual son parte los investigadores del estudio. Se diseñaron y utilizaron consentimientos informados, expresándose la finalidad del estudio, el resguardo de identidad, y la solicitud de autorización para grabar el audio de los grupos focales y las entrevistas. Todos los participantes accedieron y firmaron los consentimientos.

\section{RESULTADOS}

Los resultados presentados a continuación dan cuenta de los elementos que caracterizan la implementación de un programa público de SME, a partir de las prácticas y perspectivas de quienes lo ejecutan. Se identificaron tres categorías principales referidas a: a) la importancia de la experiencia y experticia de los ejecutores del programa, $b$ ) la adaptación en la ejecución frente a la variabilidad del contexto y c) la ejecución del trabajo que va más allá de lo indicado en las orientaciones y su traducción en instrumentos de cálculo.

En cada categoría, las citas fueron identificadas de acuerdo con el tipo de participante: para el caso de los grupos focales, se utilizó el número del enunciante y la región de pertenencia del equipo ejecutor participante del grupo focal. Para las entrevistas, se enumeraron los coordinadores y no se explicitó la región de pertenencia, con la finalidad resguardar el anonimato de los entrevistados. Al final de este apartado se ofrece una breve síntesis en torno a los hallazgos.

\section{Importancia de la experiencia y experticia en el programa}

Un aspecto central que emerge de los relatos es que el modo en que se implementa la intervención se vincula estrechamente con la experiencia acumulada por los ejecutores, que aparece como un elemento nuclear para comprender sus formas de implementación.

Así, se señala que las orientaciones estratégicas (manual) y las diferentes actividades ahí presentadas serían especialmente utiles como guía del trabajo para los profesionales que se integran al programa, pues éstos poseerían un menor conoci- 
miento de la práctica a realizar, y el manual guiaría las orientaciones de su quehacer. De hecho, se remarca el argumento de la utilidad que poseen las actividades señaladas por el instrumento de orientación estratégica, las cuales pueden ser utilizadas o descartadas en función de las experiencias previas y el conocimiento adquirido sobre su utilidad por parte de los ejecutores más antiguos:

es como tener un taller en la práctica armado, llegar y probar [...] lo nuestro tiene que ver con que llevamos mucho tiempo y hay actividades que nosotros ya de alguna forma desechamos porque ya las probamos [...] Como guía para equipos nuevos, yo creo que es una excelente opción (Ejecutora 2, grupo focal región II).

De esta manera, puede observarse que la autonomía entregada a los ejecutores, como operadores directos de la implementación del programa, permite una mayor flexibilidad para la adaptación y la resolución de problemas, a medida que existe mayor conocimiento del contexto en el que se inserta.

Asimismo, en los discursos se visibiliza la valoración positiva otorgada a las orientaciones, en relación con el acceso a intervenciones con sustento teórico: "Quizás muchas cosas nosotros ya las hacíamos, porque uno en el hacer va aprendiendo mucho, pero aquí está la teoría y una estructura" (Ejecutora 1, grupo focal región II).

Dicha valoración se potencia al considerar la concordancia que algunas de las actividades establecidas por el programa poseen con las prácticas cotidianas que los ejecutores han realizado a través del tiempo, tales como conocimientos y aprendizajes adquiridos por medio de la praxis.

Cabe señalar que existirían prácticas que escapan a lo instruido por los lineamientos del programa, como indicaciones sobre generación de vínculos, comunicación corporal, gestos o habilidades de manejo de grupo, las cuales estarían influidas por la persona con la cual se va a desarrollar la actividad (e.g. apoderados, profesores), así el interlocutor de la actividad determinará la modificación que debe ser realizada por quien ejecuta.

Se señala que, pese a la ausencia de lineamientos sobre este tipo de elementos, los equipos terminan por desarrollar dichas habilidades conforme adquieren más experiencia, y son considerados aspectos relevantes para desempeñar su labor:

hay muchos elementos que nosotros sí utilizamos y tenemos en la cabeza, pero no estaban escritos en ninguna parte, por ejemplo, la postura que tiene que tener el ejecutor al momento de realizar un taller, cuáles son las habilidades que requiere, o cómo es el abordaje que se tiene que hacer con los apoderados, o con los profesores [...] eso no existe en ninguna parte (Ejecutora 1, grupo focal región II). 
Desde quienes ejecutan y llevan más tiempo en el programa, se resalta la creatividad y el cambio necesario para que las actividades generen los efectos esperados; sobre todo en aquellas instancias donde alguna intervención no resultó según lo esperado; así, cada nueva intervención se vislumbra como una oportunidad de mejorar e innovar:

Ya cuando uno tiene más continuidad en el programa, yo creo que es porque una también busca cosas distintas, que vai' acomodando, que vai' dando vuelta, que esto no me resultó, que esto podría ir por acá, que este año ya no lo voy a plantear de esa manera, sino que voy cambiar (Ejecutora 1, grupo focal región IV).

\section{La adaptación frente a la variabilidad del contexto}

Otro aspecto que emerge reiteradamente en los relatos son las dificultades asociadas con enfrentarse a diversas realidades, tanto en términos de las entidades ejecutoras, como de la conformación de los equipos y de las características de las comunidades educativas; pues en muchas ocasiones la labor efectuada escapa a los lineamientos y directrices que pueden brindar la orientaciones técnicas. Es así como los ejecutores, utilizando la metáfora del "aterrizaje" de las actividades, dan cuenta de cómo una dificultad impulsa la necesidad de adaptación:

Es que te da ciertas orientaciones en el tema, ese es como el lado positivo, te da como los lineamientos, pero cuando buscamos aterrizar en la realidad es donde está el problema (Ejecutor 2, grupo focal región III).

Los relatos disocian lo prescrito en las orientaciones de lo realizado en el momento de la implementación en el "mundo real". Demandan adaptaciones con base en la experiencia de los ejecutores, de su conocimiento del contexto en el cual la intervención se lleva a cabo y de las posibilidades de aplicación de las modificaciones en las escuelas; las cuales muchas veces tensionan la implementación. Ejemplo de ello es la cantidad específica de estudiantes para poder realizar las actividades, número que no siempre es posible mantener. Esta es una directriz que es evaluada en los informes de avance y final que deben entregar los ejecutores, como condición para una implementación lograda, por eso la relevancia de cumplirla y la preocupación generada por su alteración.

En términos generales, los relatos valoran de manera positiva la adaptabilidad que debiese tener la intervención en SME. Por ejemplo, las adaptaciones referidas a las realidades y necesidades de los niños con quienes se trabaja, cuyas características deben ser consideradas al momento de efectuar ciertas actividades. En 
general, se señala de manera frecuente el diagnóstico de niños con hiperactividad, quienes requerirían actividades que no sólo se basen en la concentración:

Cada equipo tiene derecho a ver qué actividad hacer y qué tipo de actividad es más adecuado para el grupo que tú tienes, porque el manual no viene contextualizado para todos los niños, porque todos los niños son diferentes [...] y la gran mayoría, sino todos los nińos que trabajan en el programa con nosotros son hiperactivos y con cualquier actividad ellos se van a aburrir. Si están escuchando un cuento, se van a aburrir, si están sentados o dibujando igual (Ejecutor 2, grupo focal IX región).

Otro aspecto relevante relativo a la adaptabilidad de la intervención, se refiere al uso de los conceptos y el lenguaje empleados con los demás miembros de la comunidad educativa al momento de presentar las actividades. Esto puede ser observado en el siguiente extracto, relativo a los apoderados; se explicita que muchas veces referirse al taller como "preventivo", terminaba por generar una disposición defensiva por parte de éstos, lo cual derivó en el desarrollo de ciertas estrategias y modificaciones en los conceptos utilizados, en este caso, tornándose hacia nociones más atractivas, como "beneficiario" de la intervención:

A veces uno decía taller preventivo, como que al tiro [los apoderados] tomaban una posición como defensiva, era mejor ocupar la palabra beneficiario del taller (Grupo focal región II).

También se llevan a cabo adaptaciones producto de la escasez de tiempo con la que cuentan para poder llevar a cabo sus actividades, la cual se encuentra supeditada a diferentes voluntades, en ciertos casos, a los tiempos de los docentes y su disposición: tenemos que pedir una hora [a una profesora], decimos "un ratito", ni siquiera le decimos una hora, "un ratito, un ratito" (Ejecutora 5, Grupo focal, región V). Así, pese a que quienes ejecutan deben disponer de una cantidad mínima de tiempo (una hora), ésta no es explicitada al profesorado, y se llegan a realizar solicitudes por una pequeña parte del tiempo, en forma de petición o súplica.

Lo anterior, da cuenta de la falta de alineamiento entre los distintos actores que participan en la implementación de la intervención, pues se cruzan los objetivos y, por ende, aparece escasez de recursos para el cumplimiento de uno u otro.

Sumado a la realidad de las escuelas, se encuentran aquellas dificultades referidas a las dependencias administrativas de los equipos, en particular, a la diversidad de entidades ejecutoras de las que éstos dependen, lo cual conlleva a variaciones en la forma en que la intervención estudiada es implementada por los diferentes equipos. Como se señala, en general los equipos dependen de las municipalidades, las cuales poseen reparticiones que "aceptan" o no encargarse del programa: 
Ellos dependen de la repartición municipal que haya aceptado el programa, puede ser Cesfam, ${ }^{1}$ Departamento de Salud, Departamento de Educación o Dideco, ${ }^{2}$ tenemos esas tres modalidades, y a las Corporaciones Municipales que son privadas, que son igual que las municipalidades [...] y ellos ponen un coordinador que puede ser pagado por la Junta Nacional de Auxilio Escolar y Becas (Junaeb) o por un local y ellos tienen bajo su responsabilidad el equipo (Coordinador regional 4).

Esta heterogeneidad en la dependencia influye en un aspecto fundamental: los recursos y el modo en que éstos son obtenidos por quienes ejecutan. En este sentido, la implementación del programa, desde los ejecutores, depende de las posibilidades y voluntades financieras de cada entidad ejecutora, las que al ser municipales - gobiernos locales con autonomía presupuestaria-, generan una enorme variación entre un equipo y otro, lo cual repercute en el acceso a ciertos recursos y, por ende, a la calidad técnica de la intervención implementada:

Incide harto en cómo funciona la entidad ejecutora [...] si la entidad ejecutora no les provee materiales que ellos deberían comprar, y no se los pasan al equipo, eso va a incidir en la calidad técnica de su intervención (Coordinador regional 1).

Cabe mencionar que las diversas entidades ejecutoras de las que dependen los equipos corresponden a gobiernos locales, con diversos criterios de gestión y vaivenes políticos, que muchas veces influyen en la aceptación de la intervención desde actores relevantes o jerárquicos que, en los peores casos, termina por "estancar" la intervención. Se trata de una característica de los programas que trabajan en el sector público, respecto a las voluntades políticas y los cambios drásticos de los gobiernos locales electos durante un periodo corto de tiempo.

En términos generales, la adaptación aparece como una práctica central en el proceso de implementación programática, que es percibida positivamente, permitiendo dar mejor satisfacción a las necesidades de las escuelas. Se adaptan las actividades dependiendo del contexto de la intervención, las características de los grupos intervenidos y con base en la experiencia previa de los interventores. Esto se relaciona con la búsqueda de un mayor involucramiento por parte de la comunidad escolar y las entidades ejecutoras.

${ }^{1}$ Centro de Salud Familiar: consultorio que trabaja con el enfoque de salud familiar y comunitario, dependiente de los departamentos de salud de las municipalidades.

${ }^{2}$ Dirección de Desarrollo Comunitario: orientada al desarrollo de organizaciones comunitarias y a la concreción de proyectos de los habitantes de la comuna, para el mejoramiento de la calidad de vida, dependiente de las municipalidades. 


\section{La ejecución del trabajo y su traducción en instrumentos de cálculo}

Como se ha evidenciado previamente, seguir las orientaciones técnicas y cumplir con el desarrollo de ciertas actividades no es la única labor que se ejecuta. En efecto, a las actividades indicadas por el programa se adicionan otras acciones, las cuales son identificadas como el "real trabajo", el cual da cuenta de las dinámicas que se generan durante el tiempo de intervención con los estudiantes, donde se priorizan actividades distintas a las propuestas en las orientaciones estratégicas y que pueden implicar, por ejemplo, aprendizajes de resolución de conflictos, obediencia o respeto entre ellos:

Nos hemos ido dando cuenta de que a veces la actividad no es lo importante... me ha pasado que ha habido conflicto con los niños y la actividad se deja completamente de lado, no se alcanza a hacer la secuencia, no nos da el tiempo, pero lo que tú trabajaste entre ellos, ¡ese es el real trabajo! (Ejecutora 1, grupo focal región II).

En este sentido, la "actividad" o "secuencia" representada por las orientaciones no se ajusta a las contingencias de la ejecución, y demanda intervenciones flexibles que den respuesta a situaciones coyunturales, con una perspectiva situada.

Retomando la lógica del "real trabajo", igualmente se enfatiza la necesidad de reconocer que la labor efectuada en el programa no puede reducirse ni traducirse sólo en códigos cuantitativos que seleccionan sólo algunos aspectos de éste — cumplimiento de metas, número de sesiones, número de niños atendidos-, dejando el proceso de construcción, intervención y aprendizaje en la invisibilización y el desconocimiento:

pasa por lo que estábamos contando, es que el hecho de que todo lo que nosotros hacemos pasa a una tabla Excel, sumamente cuantitativa, reduce nuestro trabajo, o sea esa tabla Excel reduce todo lo que nosotros podamos hacer, nuestra creatividad, por mucho que seamos los pensadores del trabajo que se hace, se ve muy reducido en esa tabla, no se refleja (Ejecutora 4, grupo focal región III).

La lógica orientada a los resultados de las organizaciones públicas genera disonancias y malestar en los equipos ejecutores, debido a la traducción de su trabajo en la simplificación burocrática, en forma de instrumentos de cálculo, que no refleja su aporte y que, a la vez, tampoco los refleja a ellos. Esta invisibilización va más allá, pues se percibe que no dan cuenta del valor de su trabajo frente a otros actores, dificultando aún más su validación, principalmente en las escuelas: 
que los profesores lo vean, porque muchas veces lo que han criticado de los talleres es eso, que sienten que no hay ningún cambio para los niños, nada (Ejecutora 1, grupo focal región IX).

Sin embargo, desde los coordinadores, los informes de ejecución aparecen como un mecanismo que garantiza el cumplimiento de las actividades planificadas inicialmente, esto al permitir monitorear y evaluar características de la implementación y evitar cierta desconfianza frente al trabajo realizado por los equipos:

en el informe final se evalúa si se hizo como correspondía o no, pero igual los informes aguantan mucho, si la gente puede escribir lo que quiera, yo no estoy ahí mirando si es que hizo todo o no, en el fondo son sus conciencias (Coordinador regional 4).

Por último se destaca que los instrumentos de cálculo no sólo darían cuenta de si se ejecutó lo planificado, o lo que realmente se hizo en la intervención, sino que además evaluarían si los ejecutores han completado adecuadamente los informes. Llama la atención en este punto, que gran parte del tiempo de revisión de los informes se centre en la evaluación de cómo éstos se completan, es decir, en los propios instrumentos.

\section{Síntesis}

Inicialmente lo que caracteriza la implementación de un programa público en SME son ejecutores con niveles de experiencia diversos, lo cual implica que las orientaciones emanadas del programa a nivel central, para su correcta e ideal ejecución, se ven modificadas por la por la experticia de quienes la ejecutan. Así, se observa que a mayor experiencia y experticia, mayor es la adaptación realizada de las orientaciones; ya sea para dar respuesta a las necesidades del contexto o bien producto de evaluar la pertinencia teórica de los contenidos y su necesidad de ajuste.

Lo anterior se relaciona con el segundo punto a tener en cuenta en la implementación: la adaptabilidad. La ejecución se lleva a cabo con un alto nivel de adaptabilidad, dado principalmente por los requerimientos emanados del contexto y por llevar a cabo una intervención situada que ofrece las garantías de una intervención exitosa.

El último punto enfatiza que la ejecución del programa no sólo responde a las orientaciones técnicas, sino también que la implementación implica desarrollar otras tareas que no son visibilizadas por la intervención y que deben ser desarrolladas para su correcta ejecución. No obstante, estas otras tareas no son conside- 
radas en la intervención, pero se transforman en el "real trabajo" que sustenta la intervención. La tensión principal se produce entre la orientación a los resultados esperados del programa y su reporte mediante instrumentos de cálculo, lo cual no permitiría visibilizar las múltiples tareas que se llevan a cabo y que van más allá de lo prescrito en la intervención.

\section{DISCUSIÓN}

El propósito de este estudio fue caracterizar la implementación de un programa público SME desde las prácticas y perspectivas de quienes lo ejecutan, en el contexto de la NGP en Chile. De esta manera se distinguieron categorías que dieron cuenta de las características de los ejecutores del programa en términos de su experiencia y experticia, y la valoración que hacen de su trabajo; así como de las adaptaciones requeridas para la implementación de un programa de SME a escala nacional. Todos éstos son aspectos necesarios para comprender la implementación de un programa pensado a nivel central, pero ejecutado a nivel local.

El diseño del programa de SME estudiado opera desde una lógica de estandarizar las prácticas a través orientaciones estratégicas (guías y manuales técnicos). Para quienes se introducen en el trabajo de implementar un programa público, la estandarización se revela como un elemento que facilita y mejora la ejecución, lo cual permitiría la comparación entre procesos desde una mirada más bien homogénea (Miller, 2001).

Así, se identifica que quienes son más fieles a las orientaciones son aquellos con menos experiencia; en cambio quienes poseen más experiencia y experticia implementan mayores modificaciones al diseño. Esto podría deberse a que existe mayor conocimiento del contexto en el cual el programa se inserta. Aquellos con más experiencia serían más autónomos y presentarían una mayor flexibilidad para la adaptación de las orientaciones y maneras de ejecutar técnicamente el programa, así como una mayor y mejor resolución de problemas.

Otro aspecto central se refiere a las estrategias utilizadas por los ejecutores para responder a la variabilidad de los contextos en los cuales se implementa el programa. Bajo estas condiciones, surge un amplio rango de adaptaciones a la intervención, reflejadas principalmente en la modificación y flexibilidad de actividades según la situación, sobre todo desde los ejecutores con más experiencia en el programa, quienes son los que cuentan con mayor conocimiento del contexto de implementación, tanto político-administrativo como de características de los usuarios, lo cual es coherente con lo señalado por otros estudios (Allen et al., 2012; Leiva et al., 2020). 
El hecho de enfrentarse a realidades heterogéneas, necesariamente demanda de los ejecutores adaptaciones del programa para responder a las necesidades de los beneficiarios, lo cual da cuenta de la importancia de llevar a cabo intervenciones situadas y que respondan por una parte a los lineamientos del programa, pero también a las necesidades de quienes reciben las intervenciones (Leiva et al., 2020).

En este punto, la flexibilidad para la adaptación mencionada puede conllevar prácticas orientadas a promocionar o aumentar el involucramiento con el programa, en busca de su validación por parte de las autoridades tanto municipales como escolares, lo que implica la realización de actividades ajenas a las orientaciones estratégicas de la intervención; esto podría ser considerado como un obstaculizador, ya que el programa no se llevaría a cabo según sus objetivos, con la consiguiente baja eficiencia decisional en el nivel operativo.

Las adaptaciones en los programas serían necesarias para su correcta ejecución. Inicialmente éstas aparecen durante la fase de implementación de manera no intencionada, en respuesta a contingencias del contexto. Sin embargo, en muchas ocasiones, los cambios en las prácticas de los ejecutores se realizan premeditadamente, como un acervo de conocimiento, que forma parte de las planificaciones venideras antes de la implementación.

De hecho, la adaptación se convierte en una oportunidad de innovar y mejorar aquellas prácticas que no lograron los resultados deseados, a la vez de persistir en lo que funciona. En particular, se observan cambios en la entrega del programa y adición u omisión de nuevas actividades y etapas de la intervención. Lo anterior, con miras a mejorar la implementación, frente a la variabilidad de características y dependencias político-administrativas de cada equipo.

Pensar que la adaptación limitaría la estandarización y la fidelidad de un programa sería desconocer el contexto en el cual éstos se llevan a cabo. En efecto, no es posible imaginar una única manera de implementar un programa - a pesar de la existencia de orientaciones y lineamientos generales - y menos cuando éste ha sido creado a nivel central, pero ejecutado a nivel local con una importante diversidad cultural. En este sentido, parte de las adaptaciones son concebidas como necesarias y basadas en un conocimiento no explicitado de los lineamientos del programa, lo que implica una adición de actividades basadas en las competencias y experticia de cada ejecutor.

Por otra parte, los instrumentos de orientación estratégica (e.g. manual) aparecen como facilitadores de la implementación, entregándole sustento teórico a ésta, a la vez que permite a los ejecutores funcionar en lo operativo con la flexi- 
bilidad que se requiere para solucionar los problemas y realizar las adaptaciones necesarias para llevar a cabo la implementación del programa en contextos escolares diversos.

Lo anterior implica formas de apropiación de la intervención variadas por parte de los operadores, en función de su experiencia, y por parte de los equipos de trabajo, que generan prácticas propias para llevar adelante el programa. Esto se traduce en que estas prácticas potencialmente podrían facilitar el desempeño general de la intervención. Al ser precisamente estas prácticas las que los ejecutores reconocen como el "real trabajo"; es decir, la adaptación situada de la intervención, con la finalidad de responder a las necesidades de los usuarios, sean estos niños, profesores o apoderados.

De esta forma, el "real trabajo" no es lo prescrito, es el resultado del bagaje experiencial de cada ejecutor en relación con sus interlocutores en contexto. Por ende, las directrices, desde un nivel central, que se presentan como rígidas para una realidad local determinada, hacen necesaria la adaptación para cumplir con los objetivos de la intervención, rompiendo con lo prescrito no "aplicable a la realidad".

En cuanto a los instrumentos de cálculo, la lógica del "real trabajo" se contrapone a la lógica de la NGP en cuanto a la necesidad de generación de representaciones comunes respecto de metas (Klijn et al., 2010; Lester y Reckhow, 2013), y de la simplificación de los procesos de trabajo, convirtiéndolos en indicadores manejables para su comparación de forma homogénea. En este sentido, la traducción del proceso — el "real trabajo" - a instrumentos de medición cuantitativos invisibilizan y reducen la labor de los ejecutores.

Así, la tensión entre la cantidad y la calidad de la actividad, planteada por Soto et al. (2017), se hace patente, focalizándose la atención en una gestión para indicadores, sin considerar el impacto social de las políticas públicas (Cunill, 2009). De hecho, planteamientos de los instrumentos de orientación estratégica referentes al cumplimiento de cierto tipo de cuotas de tiempo (horas de trabajo con profesores) y de número de participantes (número mínimo y máximo de niños para trabajar en los talleres), aparecen como obstaculizadores a los ojos de los ejecutores, toda vez que se replican como directrices a ser evaluadas en los instrumentos de cálculo (informes de avance y final), dado que éstos darían cuenta de la correcta ejecución del programa.

Los instrumentos de cálculo utilizados conllevan una traducción de la implementación de la intervención, donde el proceso pasa a segundo plano, y lo principal son los resultados (Miller y O’Leary, 2007), repitiéndose el cuestiona- 
miento sobre la idoneidad y eficiencia de estos instrumentos en función de los principios de la política pública que debería enmarcar la intervención (Soto et al., 2017).

Si bien lo que se busca es simplificar procesos de trabajo multidimensionales y complejos, convirtiéndolos en indicadores manejables (Klijn et al., 2010; Lester y Reckhow, 2013), esto no se logra. De hecho, lo encontrado va en la línea de los plantedo por Pollitt (2003), quien señala que centrarse en indicadores implica una sobreconcentración en lo cuantificable (costos, número de usuarios atendidos, etc.) y una subconcentración en aspectos que no son fácilmente medibles.

De esta forma, el foco en objetivos cuantificables basados en valores, como la eficiencia y el desempeño, implican dificultades para los trabajadores públicos, que se ven obligados a dejar de lado valores compartidos de servicio, para concentrarse en la lógica económica de la gestión para resultados (Emery y Giauque, 2003; Hood, 1991; Pollitt, 2003). Esto puede complejizar la institucionalidad al disminuir la capacidad gubernamental de responder a problemas de los ciudadanos y al debilitar la coordinación entre las agencias públicas (Morales, 2014).

Por otra parte, la variabilidad respecto de las dependencias político-administrativas de cada equipo ejecutor, condicionada por el sistema público descentralizado, afecta en gran medida la calidad de las intervenciones, obstaculizándolas. Como muestran los resultados, el acceso a recursos por parte de los equipos, y la validación y apoyo que las autoridades otorguen al programa, afectarán la ejecución de diversos modos.

Este panorama da cuenta de la forma que ha tomado la descentralización en América Latina y sus consecuencias, ya mencionadas por Haefner (2000) y Salamon (2002), relativas a las dificultades que enfrentan los programas públicos para la consecución de sus objetivos, basadas en falta de coordinación y variabilidad de prácticas y énfasis de gestión. De esta forma se cuestiona la eficiencia en términos de uso de recursos y de resultados pregonados por la NGP (Hood, 1991).

Los resultados de esta investigación destacan que la complejidad del contexto público chileno, que opera bajo la NGP y la descentralización, implica que al momento de implementar un programa y en específico un programa de SME deben ser consideradas las particularidades de los ejecutores — en cuanto a su experticia y el tiempo en el programa- y de la ejecución, la cual se efectuará con altos niveles de adaptabilidad, lo que redundará en asumir un compromiso con lo estipulado por el programa y las demandas emergentes en los contextos cotidianos de ejecución. 
Los resultados y la discusión efectuada mostraron que los ejecutores deben responder a demandas locales que requieren un abordaje situado, en un mundo real con niveles contextuales de alta complejidad, como son los escenarios latinoamericanos. Esto debería ser explorado en nuevas investigaciones que articulen la implementación de programas y políticas en el concierto actual de la gestión pública. Gâ

\section{REFERENCIAS BIBLIOGRÁFICAS}

Aberbach, J.D. y T. Christensen (2001), "Radical Reform in New Zealand: Crisis, Windows of Opportunity, and Rational Actors", Public Administration, 79(2), pp. 403-422, DoI: https://doi.org/10.1111/1467-9299.00262.

Allen, J.D., L.A. Linnan y K.M. Emmons (2012), "Fidelity and Its Relationship to Implementation Effectiveness, Adaptation, and Dissemination", en R.C. Brownson, G.A. Colditz y E.K. Proctor (eds.), Dissemination and Implementation Research in HealthTranslating Science to Practice, Nueva York: Oxford University Press, pp. 281-304, Dor: https://doi.org/10.1093/acprof:oso/9780199751877.003.0014.

Atilola, O. y B. Ola (2016), “Towards School Mental Health Programmes in Nigeria: Systematic Review Revealed the Need for Contextualised and Culturally-nuanced Research", Journal of Child \& Adolescent Mental Health, 28(1), pp. 47-70, Dor: https://doi. org/10.2989/17280583.2016.1144607.

Ato, M., J.J. López-García y A. Benavente (2013), "Un sistema de clasificación de los diseños de investigación en psicología", Anales de Psicología, 29(3), Dor: https://doi. org/10.6018/analesps.29.3.178511.

Barry, M.M., A.M. Clarke, R. Jenkins y V. Patel (2013), "A Systematic Review of the Effectiveness of mental Health Promotion Interventions for Young People in Low and Middle Income Countries", BMC Public Health, 13(1), 835, Dor: https://doi.org/10.1186/14712458-13-835.

Barzelay, M. (2003), La nueva gerencia pública: Un acercamiento a la investigación y al debate de las políticas públicas, México: FCE.

Bilodeau, N., C. Laurin y A. Vining (2006), "Choice of Organizational Form Makes a Real Difference: The Impact of Corporatization on Government Agencies in Canada", Journal of Public Administration Research and Theory, 17(1), pp. 119-147, Dor: https://doi. org/10.1093/jopart/mul014.

Boyne, G.A. (1996), "Scale, Performance and The New Public Management: An Empirical Analysis of Local Authority Services", Journal of Management Studies, 33(6), pp. 809826, DoI: https://doi.org/10.1111/j.1467-6486.1996.tb00173.x.

Bryson, J.M., B.C. Crosby y L. Bloomberg (2014), "Public Value Governance: Moving Beyond Traditional Public Administration and the New Public Management", Public 
Administration Review, 74(4), pp. 445-456, Dor: https://doi.org/10.1111/puar.12238. Canales, M. (2006), Metodologías de investigación social, Santiago: Lom Ediciones.

Cejudo, G. (2016), "La fragmentación de la acción gubernamental: Intervenciones parciales frente a problemas complejos", documento de trabajo 297, CIDE, DOI: https://doi. org/10.13140/RG.2.2.27330.17607.

Chica, S. (2011), "Una mirada a los nuevos enfoques de la gestión pública", Administración \&Desarrollo, 39(53), pp. 57-74.

Chica, S. y C. Salazar (2016), "Nueva y posnueva gestión pública. ¿Continuidad o ruptura de las doctrinas de reforma a partir de 1990?”, Administración \& Desarrollo, 46(1), pp. 100-125.

Christensen, T. y Y. Fan (2018), "Post-New Public Management: A New Administrative Paradigm for China?”, International Review of Administrative Sciences, 84(2), pp. 389404, DOI: https://doi.org/10.1177/0020852316633513.

Christensen, T. y P. Lægreid (2005), "Trust in Government: The Relative Importance of Service Satisfaction, Political Factors, and Demography", Public Performance \& Management Review, DOI: https://doi.org/10.1080/15309576.2005.11051848.

Christensen, T. y P. Lægreid (2007), “The Whole-of-Government Approach to Public Sector Reform”, Public Administration Review, 67(6), pp. 1059-1066, Dor: https://doi. org/10.1111/j.1540-6210.2007.00797.x.

Christensen, T. y P. Lægreid (2008), "NPM and Beyond: Sstructure, Culture and Demography”, International Review of Administrative Sciences, 74(1), pp. 7-23, Dor: https://doi. org/10.1177/0020852307085730.

Christensen, T., P. Lægreid, P.G. Roness y K.A. Røvik (2007), Organization Theory and the Public Sector: Instrument, Culture and Myth, Londres: Routledge, Dor: https://doi. org/10.4324/9780203929216.

Christensen, T. y P. Lægreid (2010), "Introduction”, en T. Christensen y P. Lægreid (eds.), The Ashgate Research Companion to New Public Management, Farnham: Ashgate, pp. 1-13.

Cunill, N. (2009), "El mercado en el Estado", Nueva Sociedad, 221, pp. 34-53.

Denhardt, J. y R. Denhardt (2007), The New Public Service: Serving Not Steering, Nueva York: M.E.Sharpe.

Diefenbach, T. (2009), "New Public Management in Public Sector Organizations: The Dark Sides of Managerialist 'Englishtenment”, Public Administration, 87(4), pp. 892909, Dor: https://doi.org/10.1111/j.1467-9299.2009.01766.x.

Dunleavy, P., H. Margetts, S. Bastow y J. Tinkler (2011), Digital Era Governance: IT Corporations, the State, and e-Government, Nueva York: Oxford University Press, Dor: https:// doi.org/10.1093/acprof:oso/9780199296194.001.0001.

Emery, Y. y D. Giauque (2003), "Emergence of Contradictory Injunctions in Swiss NPM 
Projects", International Journal of Public Sector Management, 16(6), pp. 468-481, DoI: https://doi.org/10.1108/09513550310492111.

Fazel, M., V. Patel, S. Thomas y W. Tol (2014), "Mental Health Interventions in Schools in Low-income and Middle-income Countries", The Lancet Psychiatry, 1(5), pp. 388398, Dor: https://doi.org/10.1016/S2215-0366(14)70357-8.

Flick, U. (2007), Introducción a la investigación cualitativa, Madrid: Morata.

Forman, S.G., E.S. Shapiro, R.S. Codding, J.E. Gonzales, L.A. Reddy, S.A. Rosenfield, K.C. Ktoiber y L.M.H. Sanetti (2013), "Implementation Science and School Psychology”, School Psychology Quarterly, 28(2), pp. 77-100, Dor: https://doi.org/10.1037/ spq0000019.

González, M.M. y L. Trujillo (2008), "Reforms and Infrastructure Efficiency in Spain's Container Ports", Transportation Research Part A: Policy and Practice, 42(1), pp. 243-257, Dor: https://doi.org/10.1016/j.tra.2007.08.006.

Guerrero, O. (2019), "Neoliberalismo y neogerencia pública”, REAd, 25(2), pp. 4-21, DOI: http://dx.doi.org/10.1590/1413-2311.246.90269.

Haefner, C. (2000), "La descentralización y la planificación del desarrollo regional: ¿Ejes de la modernización de la gestión pública? Algunas notas sobre su discusión”, Revista MAD, 3, Dor: https://doi.org/10.5354/0718-0527.2011.14849.

Hood, Christopher (1991), "A Public Management for all Season?", Public Administration, 69, pp. 3-19, Dor: https://doi.org/10.1111/j.1467-9299.1991.tb00779.x.

Hsieh, H.F. y S.E. Shannon (2005), "Three Approaches to Qualitative Content Analysis", Qualitative Health Research, 15(9), pp. 1277-1288, Dor: https://doi.org/10.1177/ 1049732305276687.

Junaeb (Junta Nacional de Auxilio Escolar y Becas) (2017), Balance de gestión integral: Año 2017, disponible en: http://www.dipres.gob.cl/597/articles-172573_doc_pdf.pdf [fecha de consulta: 15 de noviembre de 2018].

Klijn, E.H., B. Steijn y J. Edelenbos (2010), "The Impact of Network Management on Outcomes in Governance Networks", Public Administration, 88(4), pp. 1063-1082, Dor: https://doi.org/10.1111/j.1467-9299.2010.01826.x.

Lee, K., K. Chun y J. Lee (2008), "Reforming the Hospital Service Structure to Improve Efficiency: Urban Hospital Specialization", Health Policy, 87(1), pp. 41-49, Dor: https:// doi.org/10.1016/j.healthpol.2007.10.003.

Leiva, L., M. George, A. Antivilo, A. Squicciarini, A. Simonsohn, B. Vargas y J. Guzmán (2015), "Salud mental escolar: Logros de una intervención preventiva en salud mental en niños y niñas del primer ciclo de enseñanza básica", Psicoperspectivas, 14(1), pp. 31-41, DOI: http://doi:10.5027/PSICOPERSPECTIVAS-VOL14-ISSUE1-FULLTEXT-508. 
Leiva, L., G. Zavala-Villalón, A. Antivilo-Bruna, B. Torres y C. Ganga-León (2020), “Implementation of a National Mental Health Intervention In Educational Communities: What do Successful Teams Do Differently?", Journal of Community Psychology, disponible en: https://doi.org/10.1002/jcop.22370

Lester, T.W. y S. Reckhow (2013), "Network Governance and Regional Equity: Shared Agendas or Problematic Partners?”, Planning Theory, 12(2), pp. 115-138, Dor: https:// doi.org/10.1177/1473095212455189.

Leyton, C. (2006), Balance del proceso de descentralización en Chile 1990-2005: Una mirada regional y municipal, Santiago: Universidad de Chile.

Marradi, A., N. Archenti y J.I. Piovani (2007), Metodología de las ciencias sociales, Buenos Aires: Emecé.

Milanesi, A. y C. Ramos (2017), "Public Management Models, Latin America”, en A. Farazmand (ed.), Global Encyclopedia of Public Administration, Public Policy, and Governance, Ámsterdam: Springer, Dor: https://doi.org/10.1007/978-3-319-31816-5_2692-1.

Miller, P. (2001), "Governing by Numbers: Why Calculative Practices Matters", Social Research, 68(2), pp. 379-396.

Miller, P. y T. O'Leary (2007), "Mediating Instruments and Making Markets: Capital Budgeting, Science and the Economy", Accounting, Organizations and Society, 32(7-8), pp. 701-734, Dor: https://doi.org/10.1016/j.aos.2007.02.003.

Morales, M. (2014), "Nueva gestión pública en Chile: Orígenes y efectos", Revista de Ciencia Politica, 34(2), Dor: https://doi.org/10.4067/S0718-090X2014000200004.

Noordegraaf, M. y T. Abma (2003), "Management by Measurement? Public Management Practices Amidst Ambiguity”, Public Administration, 81(4), pp. 853-871, Dor: https:// doi.org/10.1111/j.0033-3298.2003.00374.x.

Norẽa, A.L., N. Alcaraz-Moreno, J.G. Rojas y D. Rebolledo-Malpica (2012), "Aplicabilidad de los criterios de rigor y éticos en la investigación cualitativa”, Aquichan, 12(3), pp. 263-274.

Osborne, D. y T. Gaebler (1994), La reinvención del gobierno: La influencia del espiritu empresarial, Barcelona: Ediciones Paidos Ibérica.

Pérez, G., D. Ortiz, J.L. Zafra y L. Alcaide (2011), “De la New Public Management a la Post New Public Management: Evolución de las reformas en la gestión de las administraciones públicas españolas", Revista de Contabilidady Dirección, 13, pp. 129-150.

Pineda, J.A. y O. Orduz (2019), "Marco analítico para la gobernanza territorial: La política pública de infancia y adolescencia en Colombia”, Revista CS, 27, pp. 89-116, Dor: https:// doi.org/10.18046/recs.i27.2588.

Pollitt, C. (2003), "Joined-up Government: A Survey", Political Studies Review, 1(1), pp. 34-49, Dor: https://doi.org/10.1111/1478-9299.00004.

Pollitt, C. y G. Bouckaert (2011), Public Management Reform. A Comparative Analysis: New 
Public Management, Governance and the Neo-Weberian State, Nueva York: Oxford University Press.

Raczynski, D. (1994), "Políticas sociales y problemas de combate de la pobreza en Chile: Balance y desafíos", Colección Estudios Cieplan, 39, junio.

Ramos, C. y A. Milanesi (2018), "The Neo-Weberian State and the Neodevelopmentalist Strategies in Latin America: The Case of Uruguay", International Review of Administrative Sciences, 86(2), pp. 261-277, Dor: https://doi.org/10.1177/0020852318763525.

Rojas R., L. Leiva, B. Vargas y A.M. Squicciarini (2017), "Efectos de la fidelidad de la implementación sobre los resultados de una intervención preventiva en salud mental escolar: Un análisis multinivel”, Psychosocial Intervention, 26(3), pp. 147-154, DoI: https:// doi.org/10.1016/j.psi.2016.12.002.

Salamon, L. (2002), The Tools of Government: A Guide to the New Governance, Nueva York: Oxford University Press.

Sarno, J., A. Lyon, N. Brandt, C. Warner, E. Nadeem, S. Craig y M. Wagner (2014), “Implementation Science in School Mental Health: Key Constructs in a Developing Research Agenda", School Mental Health, 6(2), pp. 99-111.

Silverman, W.K. y S.P. Hinshaw (2008), "The Second Special Issue on Evidence-Based Psychosocial Treatments for Children and Adolescents: A 10-Year Update", Journal of Clinical Child \& Adolescent Psychology, 37(1), pp. 1-7, Dor: https://doi.org/10.1080/15374410701817725.

Soto, A., C. Fardella, A. Valenzuela y F. Carvajal (2017), "Las funciones performativas de los instrumentos en las redes de acción pública”, Psicoperspectivas: Individuo y Sociedad, 16(3), DoI: https://doi.org/10.5027/psicoperspectivas-Vol16-Issue3-fulltext-999.

Stoker, G. (2006), "Public Value Management", The American Review of Public Administration, 36(1), pp. 41-57, Dor: https://doi.org/10.1177/0275074005282583.

Van Gestel, N. y C. Teelken (2004), "Servicios de educación superior y de seguridad social en los Países Bajos: Institucionalismo y nueva gestión política”, Gestión y Politica Pública, XIII(2), pp. 427-467.

Vain, P.D. (2012), "El enfoque interpretativo en investigación educativa: Algunas consideraciones teórico-metodológicas", Revista de Educación, 3(4), pp. 37-46.

Verbeeten, F.H.M. y R.F. Speklé (2015), "Management Control, Results-Oriented Culture and Public Sector Performance: Empirical Evidence on New Public Management", Organization Studies, 36(7), pp. 953-978, DoI: https://doi.org/10.1177/0170840615580014. Volacu, A. (2018), "Justice, Efficiency, and the New Public Management", Australian Journal of Public Administration, 77(3), pp. 404-414, Dor: https://doi.org/10.1111/14678500.12263.

Waissbluth, M. y C. Arredondo (2011), Descentralización en Chile: Una trayectoria posible, Santiago: Universidad de Chile-Centro de Sistemas Públicos. 
Gloria Zavala-Villalón. Psicóloga de la Universidad de Chile y doctora en Psicología de la misma casa de estudios. Cuenta con un diplomado en metodologías cualitativas para la investigación psicosocial. Actualmente trabaja en el Departamento de Psicología de la Universidad de Chile, como profesora asistente de la línea de Psicología del Trabajo y las Organizaciones, y es coinvestigadora del proyecto Fondecyt Regular núm. 1171634, financiado por la Comisión Nacional de Investigación Científica y Tecnológica (Conicyt) de Chile. Durante su carrera profesional, se ha desempeñado en el ámbito de la psicología organizacional, psicología del trabajo y psicología comunitaria, por medio de proyectos académicos, investigativos y de consultoría. Sus intereses se abocan a la investigación centrada en el estudio de las desigualdades sociales en el mundo laboral y la implementación de intervenciones a nivel organizacional y público.

Catalina Ganga-León. Bachiller en Humanidades y Ciencias Sociales y psicóloga de la Universidad de Chile. Cuenta con un diplomado en gestión del cambio e innovación organizacional. Actualmente trabaja en el Departamento de Psicología de la Universidad de Chile, como asistente de investigación del proyecto Fondecyt Regular núm. 1171634, financiado por la Comisión Nacional de Investigación Científica y Tecnológica (Conicyt) de Chile. Durante su carrera profesional, se ha desempeñado en el ámbito de la psicología del trabajo, psicología social y psicología comunitaria, por medio de proyectos académicos, investigativos y de consultoría. Sus intereses se abocan a la investigación centrada en el estudio de las desigualdades sociales en el mundo laboral, y las problemáticas de trabajo y género.

Rodrigo Rojas-Andrade. Profesor asociado en la Escuela de Psicología de la Universidad Academia de Humanismo Cristiano. Es psicólogo de la Universidad de Tarapacá, magíster en Psicología Comunitaria, Universidad de Chile, magíster en Gestión Educacional, Universidad Andrés Bello y doctor en Psicología, Universidad de Chile. En el ámbito académico se desempeña dictando asignaturas asociadas con las metodologías de investigación social y el análisis de datos cuantitativos y cualitativos. Su principal línea de investigación es la implementación de programas psicosociales en contextos educativos y comunitarios.

Betzabé Torres Cortés. Psicóloga de la Universidad Viña del Mar y magíster(c) en Psicología Comunitaria de la Universidad de Chile. Cuenta con un diplomado en Género y Políticas Públicas, y un postítulo en Tratamiento de Adicciones, de la misma casa de estudios. Actualmente, es tesista magíster del proyecto Fondecyt Regular núm. 1171634, financiado por la Comisión Nacional de Investigación Científica y Tecnológica (Conicyt) de Chile, en el Departamento de Psicología de la Universidad de Chile. Durante su carre- 
ra profesional, se ha desempeñado en el ámbito de la psicología comunitaria, psicología clínica-comunitaria y políticas sociales, a través de docencia en educación superior, proyectos académicos, investigativos y de consultoría, y coordinación de proyectos y participación en equipos de intervención interdisciplinarios en programas públicos. Sus intereses se enfocan en la investigación centrada en el estudio de la implementación de políticas públicas con enfoque de género.

Loreto Leiva Bahamondes. Profesora asociada en el Departamento de Psicología de la Universidad de Chile. Es psicóloga de la Universidad de Chile, magíster en Psicología de la Salud, Universidad de Bordeaux, magíster en Salud Pública, Universidad de Bordeaux, y doctora en Psicología de la Pontificia Universidad Católica de Chile. Su principal interés de investigación ha sido aprender cómo las intervenciones psicosociales son capaces de promover trayectorias de salud mental positivas en contextos de alta vulnerabilidad y cómo moderar los efectos perjudiciales de los factores sociales negativos. Sus principales líneas de investigación son salud mental escolar e implementación de programas psicosociales. 